\title{
Potential contribution of erythrocyte microRNA to secondary erythrocytosis and thrombocytopenia in congenital heart disease
}

\author{
Nobuhiro Mukai ${ }^{1}$, Yoshinobu Nakayama ${ }^{1}$, Satoshi Murakami ${ }^{2}$, Toshihito Tanahashi ${ }^{3}$, Daniel I Sessler ${ }^{4}$, Sachiyo Ishii ${ }^{1}$,
} Satoru Ogawa ${ }^{1}$, Natsuko Tokuhira', Toshiki Mizobe ${ }^{1}$, Teiji Sawa ${ }^{1}$ and Yasufumi Nakajima ${ }^{5}$

BACKGROUND: Children with cyanotic heart disease develop secondary erythrocytosis and thrombocytopenia via unknown mechanisms. Mature erythrocyte microRNAs may reflect clinical pathologies and cell differentiation processes preenucleation. This study evaluated erythrocyte microRNAs in children with cyanotic heart disease.

METHODS: Erythrocyte microRNAs from children with cyanotic and acyanotic heart disease and without cardiac disease were quantified with Ion PGM System $(n=10$ per group). Differential expression was confirmed by quantitative PCR (qPCR; $n=20$ per group).

RESULTS: Mir-486-3p, mir-486-5p, and mir-155-5p increased in patients with cyanotic heart disease compared with those without heart disease: fold differences (95\% confidence interval): mir-486-3p: 1.92 (1.14-3.23), $P=0.011$; mir-486-5p: 2.27 (1.41-3.65), $P<0.001$; and mir-155-5p: 1.44 (1.03-2.03), $P=0.028$. Mir-486-5p was increased, and let-7e-5p and mir1260a were decreased in patients with acyanotic heart disease compared with those without heart disease: mir-486-5p: 1.66 (1.03-2.66), $P=0.035$; let-7e-5p: 0.66 (0.44-0.99), $P=0.049$; and mir-1260a: 0.53 (0.29-0.99), $P=0.045$.

CONCLUSION: Several microRNA levels changed in children with cyanotic and acyanotic heart disease. Mir-486-3p and -5p are associated with hematopoietic differentiation. Mir-486-3p regulates the erythroid vs. megakaryocyte lineage fate decision. Mir-155 is a hypoxia-inducible microRNA, whose overexpression inhibits megakaryocyte differentiation. Erythrocyte microRNA expression changes may contribute to erythrocytosis and thrombocytopenia in children with cyanotic heart disease.

$\mathbf{R}$ ight-to-left shunts make children with cyanotic congenital heart disease chronically hypoxemic and promote secondary erythrocytosis $(1,2)$. Erythrocytosis is mediated by renal erythropoietin secretion consequent to chronic tissue hypoxia (3). Another problem in these children is an inverse relationship between the platelet count and hematocrit (4), possibly because the shear stress due to increased blood viscosity activates platelets, thus shortening their lifespan (5). However, in a quarter of patients with cyanotic disease, reticulated platelets (an indicator of platelet production) decrease despite normal thrombopoietin concentrations, and normal platelet factor 4 and $\beta$ thromboglobulin levels suggest minimal platelet activation (4). Furthermore, after an initial increase in the circulating erythropoietin concentration, platelet production returns to normal, whereas excessive erythropoiesis is sustained (6). Thus, the pathologies of erythrocytosis and thrombocytopenia in children with cyanotic heart disease remain unclear.

MicroRNAs are small, single-stranded, functional RNA molecules composed of 18-25 nucleotides. They are transcribed from noncoding regions of genomic DNA and undergo several processes to become mature microRNAs (7). They negatively regulate gene expression with transcriptional or post-transcriptional repression (8). MicroRNAs demonstrate diverse biological activities (9-11) and may be disease-specific biomarkers (12-14).

Until recently, transcriptomic analysis of circulating erythrocytes was considered difficult because the nucleus is extruded during maturation and, thus, the cells were considered to lack genetic material. However, it is now clear that they contain abundant and diverse RNAs including microRNAs $(15,16)$. Enucleated cells are considered to inherit microRNA during the shedding process $(17,18)$ and microRNA remains in mature erythrocytes (19). Thus, microRNAs in mature erythrocytes are thought to mirror pathological processes that occur during cell differentiation.

Therefore, we tested the hypothesis that microRNAs associated with erythrocytosis and thrombocytopenia are altered in mature erythrocytes of children with cyanotic heart disease. Specially, we focused on microRNA changes in erythrocytes, and compared erythrocyte microRNA expression

${ }^{1}$ Department of Anesthesiology and Intensive Care, Kyoto Prefectural University of Medicine, Kyoto, Japan; ${ }^{2}$ Life Solution Group, Thermo Fisher Scientific Lifetechnologies, Tokyo, Japan; ${ }^{3}$ Department of Gastroenterology and Oncology, Tokushima University Graduate School of Biomedical Sciences, Tokushima, Japan; ${ }^{4}$ Department of Outcomes Research, Anesthesiology Institute, Cleveland Clinic, Cleveland, Ohio; ${ }^{5}$ Department of Anesthesiology and Intensive Care, Kansai Medical University, Osaka, Japan. Correspondence: Yoshinobu Nakayama (na-yoshi@koto.kpu-m.ac.jp) 
in children with cyanotic and acyanotic cardiac heart disease, and children without cardiac disease using next-generation sequencing technology.

\section{METHODS}

This study was approved by the Institutional Review Board of Kyoto Prefectural University of Medicine, Kyoto. Written informed consent was obtained from the parents or guardians of participating infants and children.

We enrolled infants and children $<5$ years old who were scheduled for elective surgery at Kyoto Prefectural University of Medicine from November 2015 to February 2017. The cyanotic and acyanotic groups were restricted to those scheduled for cardiac surgeries, and the control group was restricted to those scheduled for minor surgeries (e.g., inguinal hernioplasty) with American Society of Anesthesiologists physical status 1. Patients with chromosomal abnormalities (e.g., Down's syndrome and 22q11.2 deletion) or inflammatory disease and those who had been administered blood products within the last 3 months were excluded. Patients with significant right-to-left shunts at more than 3 months were enrolled as a cyanotic group. We excluded cyanotic patients younger than 3 months because they were possibly within the lifespan of their original erythrocytes. Patients with significant left-to-right shunts (pulmonary/systemic blood flow >1.5) were enrolled as an acyanotic group.

In an initial exploratory phase, comprehensive analyses with nextgeneration sequencing technology were performed using blood from children with cyanotic $(n=10)$ and acyanotic $(n=10)$ heart disease and without cardiac disease (control group, $n=10$ ). In the subsequent validation phase, changes in microRNAs identified in the initial phase were verified with the quantitative PCR (qPCR) in 20 patients per group.

\section{Sample Preparation}

Whole blood samples $(2.7 \mathrm{ml})$ were taken from the arterial monitoring catheter immediately after anesthetic induction before surgery. The samples were collected with $0.3 \mathrm{ml}$ of sterile $4.0 \%$ citrate concentrated solution (Sigma-Aldrich, St. Louis, MO) in a Falcon tube. Briefly, blood samples were centrifuged $(200 \mathrm{~g} \times 25 \mathrm{~min}$ at room temperature), and the plasma and buffy coat were removed. The sample was resuspended to a $\mathrm{Ht}$ of $30-40 \%$ with Dulbecco's phosphate-buffered saline without calcium or magnesium chloride, and gravity filtered to remove leukocytes (LeukoCatch II, WATSON, Tokyo, Japan). The complete blood count was measured using MYTHIC 22OT (J) (A\&T Corporation, Kanagawa, Japan).

\section{MicroRNA Isolation and Next-Generation Sequencing (Exploratory Phase)}

MicroRNA was extracted from blood cells of patients using the RNeasy Plus Mini Kit and RNeasy MinElute Cleanup Kit (Qiagen, Venlo, the Netherlands) according to the manufacturer's instructions. The number of each patient's blood cells was aligned to $3.0 \times 10^{8}$ cells using the complete blood count. The microRNA size range was confirmed and $2 \mathrm{ng}$ of RNA was quantified using Bioanalyzer and the Agilent Small RNA kit (Agilent Technologies, Santa Clara, CA).

Complementary DNA libraries were constructed using the Ion Torrent RNA-seq kit v.2 (ThermoFisher Scientific, Waltham, MA) for small RNA libraries according to the manufacturer's instructions, and their purity and concentration were confirmed using Bioanalyzer with the Agilent DNA kit (Agilent Technologies).

MicroRNA library sequencing was performed on a 318 chip using an Ion Personal Genome Machine system (ThermoFisher Scientific). All raw reads were automatically trimmed to remove adaptors, aligned on a human genome (hg19 GRCh37), and annotated using Torrent Suite 1.5 (TMAP) with default parameters. Small and noncoding RNAs were classified according to gene types. We identified sequences with the miRBase 21 (http://www/mirbase.org/) microRNA database.

\section{Total RNA Isolation and qPCR (Validation Phase)}

We performed qPCR to determine mature microRNA and validate the results of next-generation sequencing. Total RNA was extracted from $2.7 \mathrm{ml}$ of patients' blood cells using RNeasy Plus Mini Kit and RNeasy MinElute Cleanup Kit (Qiagen), according to the manufacturer's instructions. The RNA was quantified from $1.0 \times 10^{8}$ cells using Quantus Fluorometer (Promega KK, Tokyo, Japan), and the concentration of each sample was $0.2 \mathrm{ng} / \mu \mathrm{l}$. Small RNA molecules, 5'-UUUGGAUUGAAGGGAGCUCUA-3', were spiked into samples and used as a spike-in control.

Complementary DNA was produced using the ABI Taqman advanced microRNA complementary DNA synthesis kit (ThermoFisher Scientific). qPCR analysis was conducted using Step One Plus (ThermoFisher Scientific). Forty-cycle amplification (Ct values $=40$ ) was used for the cycle threshold. We excluded microRNA from group comparisons when undetermined samples (Ct values $>40$ ) exceeding three samples were seen in a group. The $\Delta \Delta \mathrm{Ct}$ method was used to analyze the expression values.

\section{Statistical Analysis}

Empirical analysis of digital gene expression using CLC Genomic Workbench version 8.5.1 (Qiagen) was applied to compare the

Table 1. Differentially expressed microRNAs in three groups compared using next-generation sequencing

\begin{tabular}{|c|c|c|c|c|c|c|}
\hline \multirow[t]{2}{*}{ MicroRNA ID } & \multicolumn{2}{|c|}{ Control vs. cyanotic } & \multicolumn{2}{|c|}{ Control vs. acyanotic } & \multicolumn{2}{|c|}{ Cyanotic vs. acyanotic } \\
\hline & $\log _{2}$ fold change & FDR- $P$ value & $\log _{2}$ fold change & FDR- $P$ value & $\log _{2}$ fold change & FDR- $P$ value \\
\hline mir-486-1 & 4.79 & 0.0002 & 5.15 & $2.11 E-05$ & 0.36 & 1 \\
\hline mir-629 & 3.8 & 0.0007 & 0.17 & 1 & -3.62 & 0.0006 \\
\hline mir-155 & 2.47 & 0.005 & -0.79 & 1 & -3.26 & 0.0002 \\
\hline mir-1260a & 1.19 & 1 & -5.95 & $3.53 E-05$ & -7.14 & $1.76 E-06$ \\
\hline mir-1260b & 0.86 & 1 & -6.14 & $2.66 E-05$ & -7.0 & $1.76 E-06$ \\
\hline mir-296 & 1.96 & 1 & -4.91 & 0.016 & -4.53 & $8.106 E-05$ \\
\hline mir-7977 & 5.55 & 1 & -0.72 & 1 & -6.27 & 0.028 \\
\hline
\end{tabular}

FDR, false-discovery rate.

The microRNAs were recognized as stem-loop sequences. For mir-1260a and mir-1260b, the stem-loop and mature sequences showed a one-to-one correspondence. 


\section{Articles | Mukai et al.}

results of microRNA expression from next-generation sequencing. False-discovery rate-corrected $P$ values (FDR- $P$ values) less than 0.05 were considered significant in the analysis.

The expression values of qPCR were compared by one-way analysis of variance followed by Tukey's post hoc test. Three-group variables were subjected to a normality test to determine data distributions. Normally distributed data are expressed as the means (SD), and non-normally distributed data are expressed as the median with the first and third quartiles (Quartile 1 and Quartile 3). The variables were analyzed using Tukey's multiple-comparison test for normally distributed data and Dunn's test for non-normally distributed data. $P$ values less than 0.05 were considered significant. Statistical analyses were performed with Stat-Flex version 6.0 (Artech, Osaka, Japan) and Graph Pad Prism 7.00 (Graphpad Software, La Jolla, CA).
We estimated that 10 patients per group were an appropriate sample size for the exploratory phase based on a previous study $(20,21)$. Sample size estimation for the qPCR validation phase was based on $\alpha=0.05, \beta=0.2$, and effect size $=0.5$, with three groups, using $G^{*}$ power 3.1.9.2 (http://www.gpower.hhu.de/). Fourteen patients per group were required. Thus, we enrolled 20 patients per group.

\section{RESULTS}

Next-Generation Sequencing (Exploratory Phase)

We isolated and analyzed microRNA from 30 pediatric patients. The characteristics of the patients are shown in Supplementary Table S1 online. Five patients were at the stage of post-palliative surgeries in the cyanotic group: a

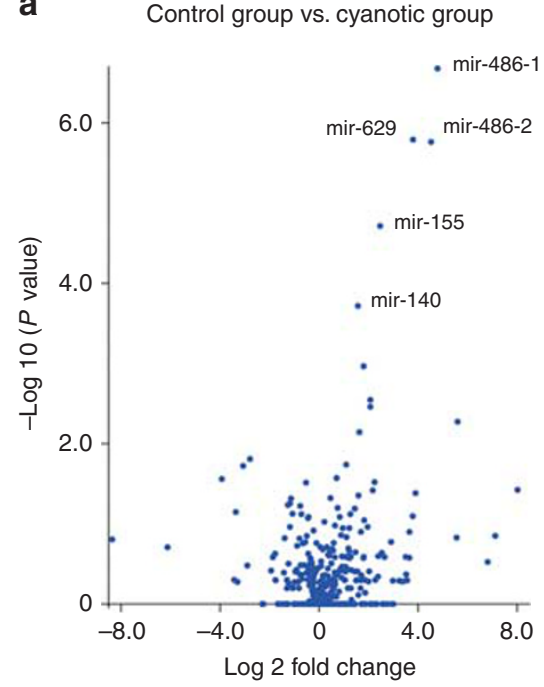

Control group vs. acyanotic group

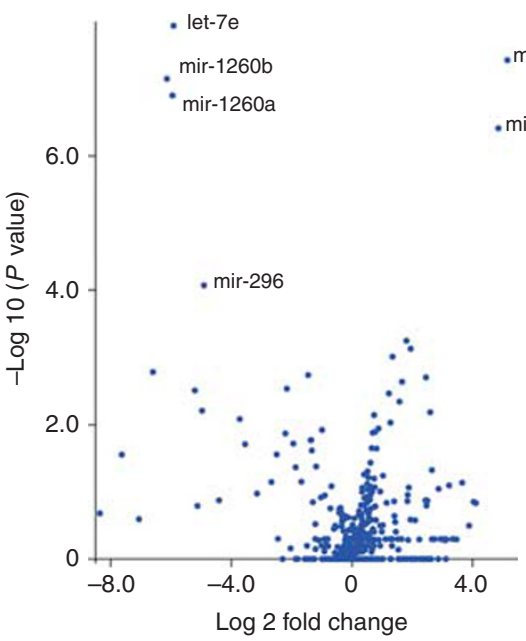

Cyanotic group vs. acyanotic group

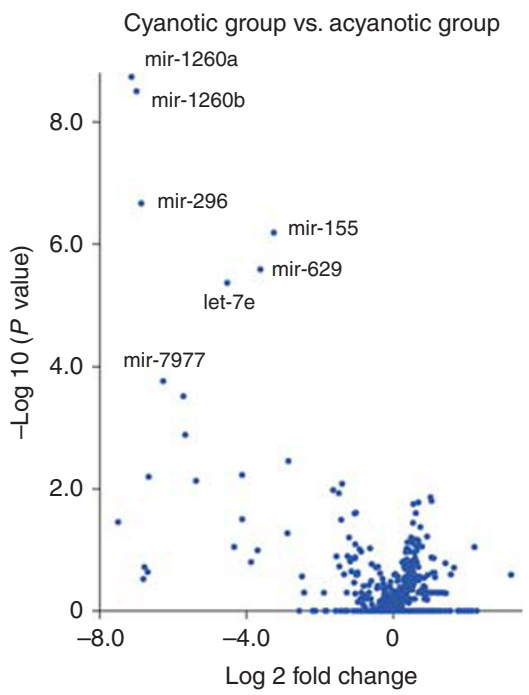

b

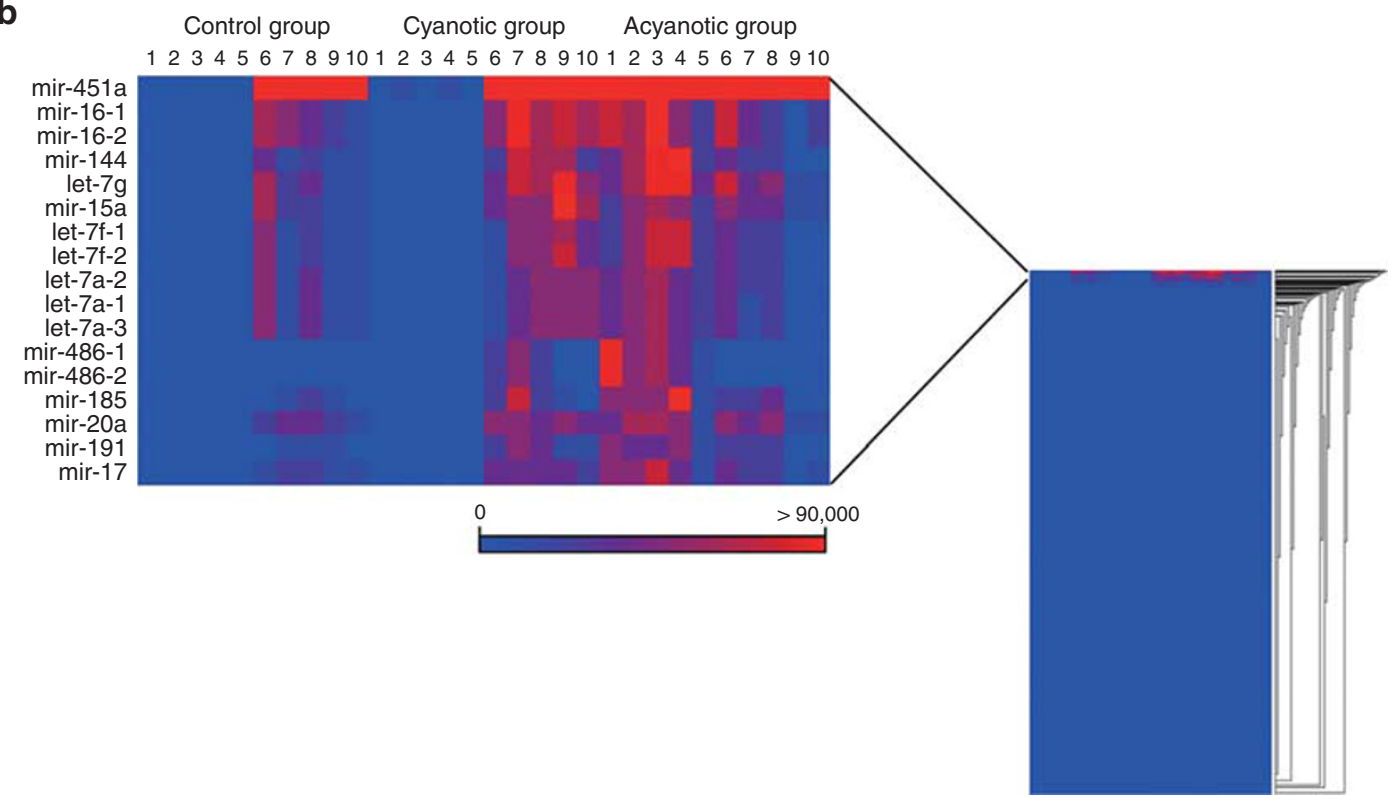

Figure 1. Volcano plot of each group comparison and a heat map of the three groups. (a) The names of microRNAs with FDR-corrected $P$ values (FDR- $P$ values) less than 0.05 are presented. The microRNAs were recognized as stem-loop sequences. For mir-1260a and mir-1260b, the stem-loop and mature sequences showed a one-to-one correspondence. The microRNA database used was miRBase 21 (http://www/mirbase.org/). (b) A whole heat map is presented on the right. An area of high expression is expanded on the left. Mir-486-1 and mir-486-2 clearly show a contrast in the cyanotic congenital heart disease and acyanotic congenital heart disease groups vs. control group. FDR, false-discovery rate. 
Tricuspid stenosis (post-Glenn procedure), hypoplastic left heart syndrome (post-pulmonary artery banding procedure), pulmonary atresia (post-Blalock-Taussig shunt procedure), hypoplastic left heart syndrome (post-Norwood procedure), and tricuspid atresia (post-Glenn procedure). The resting oxygen saturation $\left(\mathrm{rSPO}_{2}\right)$ in the cyanotic group was $81 \pm 7 \%$. Eight patients had ventricular septal diseases in the acyanotic group. Hemoglobin $(\mathrm{Hb})$ and hematocrit $(\mathrm{Ht})$ in the cyanotic group were significantly higher than in the other groups, and the platelet count (PLT) in the cyanotic group was lower than in the control group. The 10 microRNAs exhibiting significant differences on three-group comparison are shown in Table 1. A volcano plot of each group comparison and heat map are shown in Figure 1. The expression of mir-486 was markedly increased in both cyanotic and acyanotic groups vs. the control group. The expression of mir-155 was significantly increased in the cyanotic vs. control group. The expressions of mir-1260a, mir-1260b, and let-7e were significantly decreased in only the acyanotic group.

\section{qPCR (Validation Phase)}

We isolated and analyzed the significant microRNAs identified in the initial phase from 60 pediatric patients. The characteristics of patients are shown in Supplementary Table S2. Nine patients were at the stage of post-palliative surgeries in the cyanotic group: tricuspid stenosis (postpulmonary artery-banding procedure, $n=2$ ), hypoplastic left heart syndrome (post-Norwood procedure), pulmonary atresia (post-Blalock-Taussig shunt procedure), left single ventricular (post-pulmonary artery-banding procedure), right single ventricular (post-Glenn procedure), right single ventricular (post-Blalock-Taussig shunt procedure, $n=2$ ), and tricuspid atresia (post-Glenn procedure). The $\mathrm{rSPO}_{2}$ in the cyanotic group was $83 \pm 9 \%$. Eleven ventricular septal disease patients were in the acyanotic group. The erythrocyte count, $\mathrm{Hb}$, and $\mathrm{Ht}$ in the cyanotic group were significantly higher than in the other groups, and PLT in the cyanotic group was lower than in the control group. The results of three-group comparison are shown in Table 2, and the five mature microRNAs showing significant differences are presented in Figure 2. Mir-486-3p, mir-486-5p, and mir$155-5 p$ were significantly increased in the cyanotic vs. control group. Mir-486-5p was significantly increased, and let-7e-5p and mir-1260a were significantly decreased in the acyanotic vs. control group. Mir-486-3p was also higher in the cyanotic than in the acyanotic group, and there was no significant difference between acyanotic and control groups.

\section{DISCUSSION}

We compared microRNA changes in erythrocytes from children with cyanotic heart disease, acyanotic heart disease, and without heart disease based on comprehensive analyses using next-generation sequencing technology and qPCR to identify potential mechanisms of secondary erythrocytosis and thrombocytopenia in children with cyanotic heart disease. Several microRNA expressions were significantly changed in children with both cyanotic and acyanotic heart disease. Specifically, mir-486-3p, mir-486-5p, and mir-155-5p may contribute to erythrocytosis and thrombocytopenia in cyanotic heart disease.

Both mir-486-3p and mir-486-5p are closely associated with hematopoietic differentiation. The mir-486-3p megakaryocyte axis, especially, regulates the erythroid vs. megakaryocyte lineage fate decision (22). Mir-486-3p is upregulated by the transactivation of ankyrin-1 gene expression and it downregulates musculoaponeurotic fibrosarcoma proto-oncogene protein coding gene mRNA. Through its downregulation, the myeloblastosis proto-oncogene protein coding gene inhibits megakaryocyte commitment and induces megakaryocyte erythroid progenitor differentiation into erythroblasts. Elevation of mir-486-3p may thus contribute to erythrocytosis and thrombocytopenia in children with cyanotic heart disease $(4,5)$.

Mir-486-5p, which was elevated in children with both cyanotic and acyanotic heart disease, enhances the differentiation of normal CD $34^{+}$cells (upstream of hematopoietic

Table 2. Differentially expressed microRNAs in three groups compared using qPCR

\begin{tabular}{|c|c|c|c|c|c|c|}
\hline \multirow[t]{2}{*}{ MicroRNA ID } & \multicolumn{2}{|c|}{ Control vs. cyanotic } & \multicolumn{2}{|c|}{ Control vs. acyanotic } & \multicolumn{2}{|c|}{ Cyanotic vs. acyanotic } \\
\hline & Fold change $(95 \% \mathrm{Cl})$ & $P$ value & Fold change $(95 \% \mathrm{Cl})$ & $P$ value & Fold change $(95 \% \mathrm{Cl})$ & $P$ value \\
\hline mir-486-3p & $1.92(1.14-3.23)$ & 0.011 & $1.09(0.64-1.83)$ & 0.92 & $0.57(0.34-0.95)$ & 0.029 \\
\hline let-7e-5p & $0.99(0.66-1.49)$ & 0.99 & $0.66(0.44-0.99)$ & 0.049 & $0.67(0.45-1.0)$ & 0.055 \\
\hline mir-1260a & $0.89(0.48-1.65)$ & 0.89 & $0.53(0.29-0.99)$ & 0.045 & $0.6(0.32-1.11)$ & 0.12 \\
\hline mir-140-3p & $1.17(0.75-1.81)$ & 0.69 & $1.28(0.82-1.99)$ & 0.38 & $1.1(0.7-1.71)$ & 0.87 \\
\hline mir-629-3p & $1.32(0.66-2.64)$ & 0.61 & $0.8(0.41-1.58)$ & 0.72 & $0.61(0.3-1.23)$ & 0.22 \\
\hline mir-629-5p & $1.56(0.76-3.22)$ & 0.3 & $1.21(0.59-2.47)$ & 0.79 & $0.77(0.37-1.61)$ & 0.68 \\
\hline
\end{tabular}

95\% Cl: $95 \%$ confidence interval; qPCR, quantitative PCR.

Mir-296-5p and let-7e-3p are not shown because they were undetected. Mir-155-3p, mir-140-5p, mir-296-3p, and mir-7977 are not shown because they were undetermined (Ct values $>40)$. 

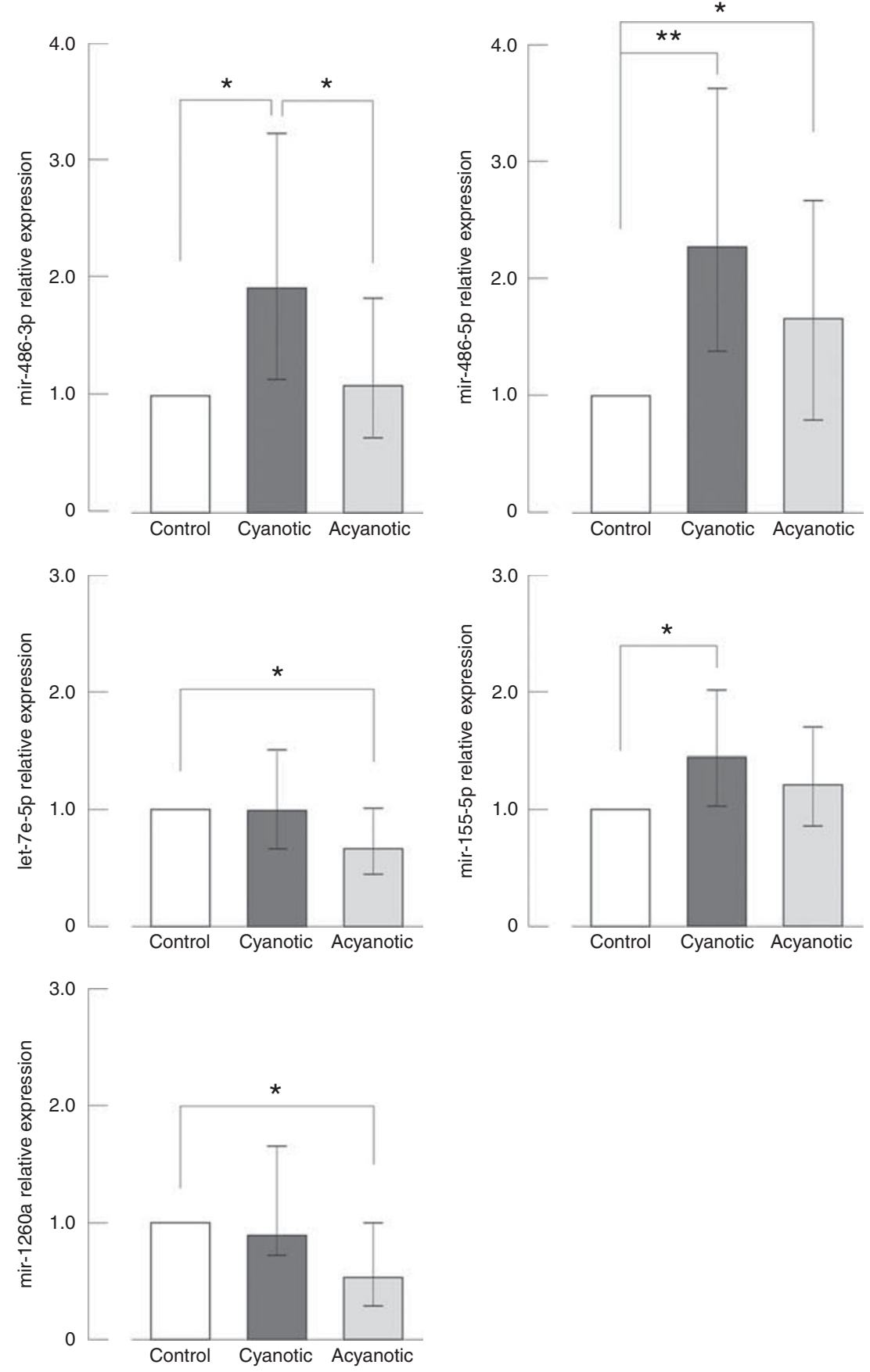

Figure 2. The differential microRNAs in the three-group comparison validated by $q P C R$. Mir-486-3p, mir-486-5p, and mir-155-5p were significantly increased in the cyanotic congenital heart disease group. ${ }^{*} P<0.05$, ${ }^{*} P<0.01$. The error bars represent the $95 \%$ confidence interval. qPCR, quantitative PCR.

differentiation) and inhibits the suppression of normal CD34 ${ }^{+}$ cells in vitro and in vivo (23). Increased mir-486-5p is thought to be a mechanism compensating for chronic tissue hypoxia by increasing circulating hemocytes. Children with acyanotic heart disease did not have right-to-left shunts, but often suffered from chronic tissue hypoxia due to systemic hypoperfusion caused by left-to-right shunts. Therefore, increases in both circulating hemocytes and the blood volume may be more advantageous than the compensatory mechanism of mir-486-3p and mir-155-5p elevation (downstream of hematopoietic differentiation) in the presence of acyanotic congenital heart disease. Consequently, it is consistent with the trend, whereby children with acyanotic heart disease generally do not develop erythrocytosis or thrombocytopenia (4).

Mir-155 is among the hypoxia-inducible microRNAs $(24,25)$. It modulates megakaryopoiesis, and its overexpression inhibits the differentiation of megakaryocytes by 
regulating Meis-1 and Ets-1 transcription factors (26). Overexpression of mir-155 may therefore explain erythrocytosis and thrombocytopenia in children with cyanotic heart disease. Mir-155 is also known to regulate hexokinase II, and its elevation promotes hexokinase II and protein production (27). Anaerobic glycolysis mainly provides erythrocytes with energy because mitochondria are removed from reticulocytes during the final stage of erythrocyte differentiation (28).

It is interesting that mir-155 not only facilitates erythrocytosis, but also anaerobic glycolysis in erythrocytes, which, in turn, promotes adaptation to hypoxia. Moreover, mir-155 is expressed in lymphocytes, endothelial cells, and smooth muscle cells (29-31). Recently, mir-155 was shown to possess both anti-angiogenic and pro-arteriogenic functions, via the angiotensin II type 1 receptor in endothelial cells and suppressor of cytokine signaling 1 receptor in macrophages (32). When children with cyanotic heart disease develop collateral circulation, such as the major aortopulmonary collateral artery, mir-155 may facilitate neovascularization.

Although explanations have been proposed for cyanotic heart disease pathologies, the mechanism remains unclear (4,33-35). The mechanism associated with microRNAs involving mir-486-3p, mir-486-5p, and mir-155-5p may be the most appropriate explanation to date. This is supported by the fact that the decreased reticulated platelet count in cyanotic congenital heart disease, despite the normal thrombopoietin level (4) and increased erythropoiesis, is sustained after an initial rise in erythropoietin levels (6). In mice, it was recently suggested that the lung is another primary site of terminal thrombocytic production where megakaryocytes migrate out of the bone marrow and release thrombocytes (36). Therefore, right-to-left shunt induces megakaryocyte "shunt" and may accelerate thrombocytopenia (Figure 3).

Previous studies indicated that chronic hypoxia inhibits the expression and activity of Dicer, which processes precursor microRNAs into their mature forms (37-39). Thus, the expression of mature microRNAs generally decreased with the accumulation of pre-microRNAs under chronic hypoxia. Inversely, both mir-155 and pre-mir-155 increase with hypoxia and knockdown of Dicer (27); mir-486-3p and $-5 p$ may have behaved similarly in the present study. Residual Dicer appears to preferentially process such pre-microRNAs, otherwise their degradation would be delayed under hypoxia, but the detailed mechanism remains unclear (27).

It also remains unclear whether mir-1260a and let-7e-5p, which were decreased in children suffering from acyanotic heart disease, contribute to blood cell differentiation. The downregulation of circulating let-7e-5p was identified in ventricular septal defect patients, suggesting that it plays a regulatory role in the development of ventricular septal defect (40). Most acyanotic group subjects were ventricular septal defect patients in the present study, and the downregulation of let-7e-5p might also be observed in erythrocytes. We have summarized the genes associated with blood cell

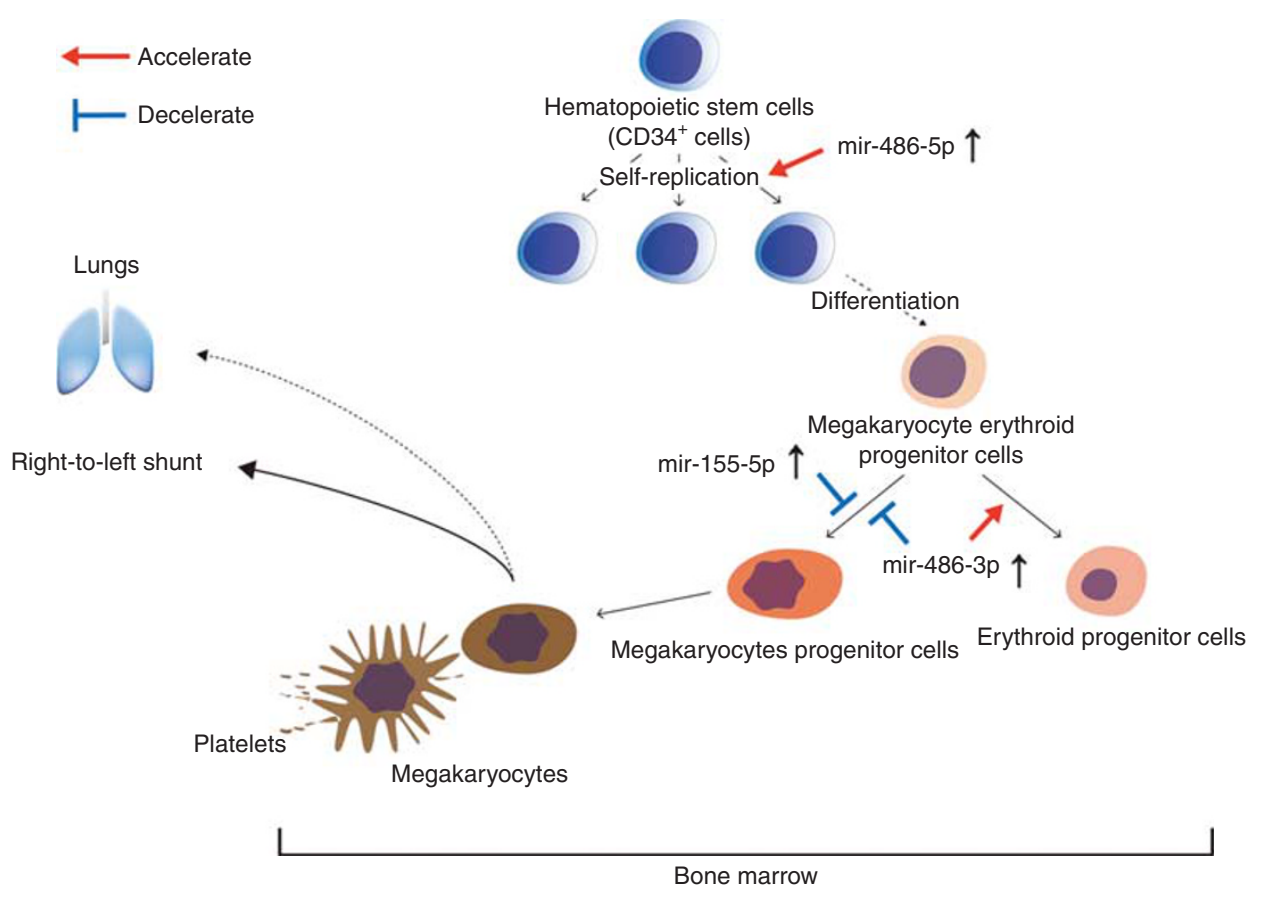

Figure 3. Schema of the possible mechanism of blood abnormalities in congenital heart disease. The elevation of mir-486-5p enhances the differentiation of $\mathrm{CD}_{3} 4^{+}$cells (upstream of hematopoietic differentiation). Elevations of mir-486-3p and mir-155-5p induce the differentiation of megakaryocyte erythroid progenitor cells into erythroid progenitor cells (downstream of hematopoietic differentiation). Right-to-left shunt induces megakaryocyte "shunt" and may accelerate thrombocytopenia(36). 
Table 3. Putative target genes based on differentially expressed microRNAs in the present study

\begin{tabular}{|c|c|}
\hline MicroRNA ID & Putative target genes \\
\hline $\operatorname{mir}-486-3 p$ & LIN54, USP42, ALCAM, BCLAF1, PRDM4, SWAP70, LIN7C, NXN, CBX5, GFAP, SLC9A8, FAM81A, and MAF \\
\hline mir- & $\begin{array}{l}X C L 16, \text { EREG, FOXO1, LIN54, PRDM4, KIF1B, LAMP3, SWAP70, LIN7C, RAB18, CYP7A1, KIF16B, MAT2A, NXN, CBX5, SLC9A8, ZNRF2, FAM81A, } \\
\text { nd RAB14 }\end{array}$ \\
\hline mir-1260a & TSPAN12, EREG, CDKN2B, LIN54, ALCAM, BCLAF1, SLC11A2, PRDM4, KIF1B, SWAP70, LIN7C, LAMP3, TRIB3, CBX5, KCNJ2, GFAP, and RAB14 \\
\hline let-7e & TSPAN12, FOXO1, ALCAM, SLC11A2, KIF1B, AKT3, LAMC1, TAF9B, RAB18, MAT2A, CBX5, KCNJ2, FAM81A, and RAB14 \\
\hline mir-155 & $\begin{array}{l}\text { HMGCR, MAP3K8, EREG, CDKN2B, FOXO1, LIN54, ALCAM, BCLAF1, SLC11A2, KIF1B, LAMP3, SWAP70, LIN7C, AKT3, LAMC1, TAF9B, MYLIP, } \\
\text { RAB18, CYP7A1, KIF16B, NXN, CBX5, KCNJ2, SLC9A8, ZNRF2, RAB14, and MAF }\end{array}$ \\
\hline \multicolumn{2}{|r|}{$\begin{array}{l}\text { AKT3, v-akt murine thymoma viral oncogene homolog 3; ALCAM, activated leukocyte cell adhesion molecule; BCLAF1, BCL2-associated transcription factor 1; CBX5, chromobox } \\
\text { homolog 5; CDKN2B, cyclin-dependent kinase inhibitor 2B; CXCL16, chemokine (C-X-C motif) ligand 16; CYP7A1, Cytochrome P450; EREG, epiregulin; FAM81A, family with } \\
\text { sequence similarity 81, member A; FOXO1, forkhead box O1; GFAP, glial fibrillary acidic protein; HMGCR, 3-hydroxy-3-methylglutaryl-CoA reductase; KCNJ2, potassium inwardly } \\
\text { rectifying channel, subfamily J, member 2; KIF1B, kinesin family member 1B; KIF16B, kinesin family member 16B; LAMP3, lysosomal-associated membrane protein 3; LAMC1, lami- } \\
\text { nin, gamma 1; LIN7C, lin-7 homolog C; LIN54, lin-54 homolog; MAF, v-maf musculoaponeurotic fibrosarcoma oncogene homolog; MAP3K8, mitogen-activated protein kinase 8; } \\
\text { MAT2A, methionine adenosyltransferase II, alpha; MYLIP, myosin regulatory light chain-interacting protein; NXN, nucleoredoxin; PRDM4, PR domain containing 4; SLC9A8, solute } \\
\text { carrier family 9; SLC11A2, solute carrier family 11, member 2; SWAP70, SWAP switching B-cell complex 70-kDa subunit; TAF9B, TAF9B RNA polymerase II; TRIB3, tribbles homolog } \\
\text { 3; TSPAN12, tetraspanin 12; USP42, ubiquitin specific peptidase 42; ZNRF2, zinc and ring finger 2. } \\
\text { The target genes were predicted based on microRNA.org (http://Www.microrna.org/). }\end{array}$} \\
\hline
\end{tabular}

differentiation that are putative targets of microRNAs to promote further studies (Table 3 ).

A limitation of this study is that it was purely observational. We did not perform functional analyses of the significant microRNAs to provide direct evidence of the blood abnormalities occurring in cyanotic patients. Further studies are therefore needed to clarify the complex mechanism of microRNA combination. Mir-155-3p, mir-140-5p, and mir296 (-3p and $-5 p)$ were excluded from three-group comparison of qPCR for statistical accuracy because several samples had undetermined $\mathrm{Ct}$ values ( $\mathrm{Ct}$ values $>40$ ). It might be more favorable to change the $\mathrm{Ct}$ value threshold for the detection of these microRNAs. For example, nearly half of the samples of mir-140-5p in control and acyanotic groups did not reach the threshold, although an amplification curve was observed. This might be because the mir-140-5p level was generally low and it was difficult to detect under nonpathological conditions. For such microRNAs, next-generation sequencing might help identify trace alterations.

An additional limitation is that the duration and extent of cyanosis differed among participating cyanotic patients, which might influence the production of microRNAs. Actually, the degrees of thrombocytopenia and erythrocytosis were not severe in our cyanotic group. This may be because the surgical interventions were performed earlier than those previously reported $(4,33,34,41)$, thus limiting the duration of chronic hypoxia, which is consistent with the unremarkable elevation of mir-155, hypoxia-inducible microRNA, both in next-generation sequencing and qPCR. In addition, the earlier surgical interventions might result in the patients included in the cyanotic and acyanotic groups being younger than those in the control group. Moreover, mir-486-5p showed a significant change in children suffering from cyanotic heart disease. The increasing normal $\mathrm{CD} 34^{+}$cells and erythroid differentiation by mir-486-5p may mask the effect of inhibiting the differentiation of megakaryocytes by mir-486$3 p$ and mir-155-5p. Finally, reticulocytes were not removed in the present study, although the numbers were similar in all three groups.

In conclusion, expression levels of several microRNAs in erythrocytes were significantly increased or decreased in children with cyanotic and acyanotic heart diseases. Several forms, including mir-486-3p, mir-486-5p, and mir-155-5p, may contribute to the development of secondary erythrocytosis and thrombocytopenia in cyanotic children following hypoxia.

\section{AUTHOR CONTRIBUTIONS}

N.M. and Yo.N.: study design, patient recruitment, data collection, data analysis, and writing the initial draft of the manuscript; S.M., T.M., T.S., and Ya.N.: study design, data interpretation, and writing the initial draft of the manuscript; T.T.: study design and writing the initial draft of the manuscript; D.I.S.: study design (supervising statistical approach) and revising the manuscript critically for important intellectual content; S.I., S. O., and N.T.: patient recruitment, data collection, and writing the initial draft of the manuscript.

\section{STATEMENT OF FINANCIAL SUPPORT}

This work was partially supported by Grants-in-Aid for Scientific Research from the Japanese Ministry of Education, Culture, Sports, Science, and Technology (Nos. 26462370, 15K21287, 15K10542, and 17K11093).

Disclosure: The authors declare no conflict of interest.

\section{REFERENCES}

1. Verel D. Blood volume changes in cyanotic congenital heart disease and polycythemia rubra vera. Circulation 1961;23:749-53.

2. Rosenthal A, Button LN, Nathan DG, Miettinen OS, Nadas AS. Blood volume changes in cyanotic congenital heart disease. Am J Cardiol 1971;27:162-7.

3. Gidding SS, Stockman JA 3rd. Erythropoietin in cyanotic heart disease. Am Heart J 1988;116:128-32.

4. Lill MC, Perloff JK, Child JS. Pathogenesis of thrombocytopenia in cyanotic congenital heart disease. Am J Cardiol 2006;98:254-8.

5. Horigome H, Hiramatsu Y, Shigeta $O$, Nagasawa T, Matsui A. Overproduction of platelet microparticles in cyanotic congenital heart disease with polycythemia. J Am Coll Cardiol 2002;39:1072-7.

6. Haga P, Cotes PM, Till JA, Minty BD, Shinebourne EA. Serum immunoreactive erythropoietin in children with cyanotic and acyanotic congenital heart disease. Blood 1987;70:822-6. 
7. Lee Y, Ahn C, Han J, et al. The nuclear RNase III Drosha initiates microRNA processing. Nature 2003;425:415-9.

8. Bartel DP. MicroRNAs: genomics, biogenesis, mechanism, and function. Cell 2004;116:281-97.

9. Lee DY, Deng Z, Wang CH, Yang BB. MicroRNA-378 promotes cell survival, tumor growth, and angiogenesis by targeting SuFu and Fus-1 expression. Proc Natl Acad Sci USA 2007;104:20350-5.

10. Kahai S, Lee SC, Lee DY, et al. MicroRNA miR-378 regulates nephronectin expression modulating osteoblast differentiation by targeting GalNT-7. PLoS One 2009;4:e7535.

11. Viticchie G, Lena AM, Latina A, et al. MiR-203 controls proliferation, migration and invasive potential of prostate cancer cell lines. Cell Cycle 2011;10:1121-31.

12. Bonci D, Coppola V, Patrizii M, et al. A microRNA code for prostate cancer metastasis. Oncogene 2016;35:1180-92.

13. Mitchell PS, Parkin RK, Kroh EM, et al. Circulating microRNAs as stable blood-based markers for cancer detection. Proc Natl Acad Sci U.S.A 2008;105:10513-8.

14. Blennow K, Dubois B, Fagan AM, Lewczuk P, de Leon MJ, Hampel H. Clinical utility of cerebrospinal fluid biomarkers in the diagnosis of early Alzheimer's disease. Alzheimers Dement 2015;11:58-69.

15. Rathjen T, Nicol C, McConkey G, Dalmay T.. Analysis of short RNAs in the malaria parasite and its red blood cell host. FEBS Lett 2006;580: 5185-8.

16. Chen SY, Wang Y, Telen MJ, Chi JT. The genomic analysis of erythrocyte microRNA expression in sickle cell diseases. PLoS ONE 2008;3:e2360.

17. Opalinska JB, Bersenev A, Zhang Z, et al. MicroRNA expression in maturing murine megakaryocytes. Blood 2010;116:e128-38.

18. Sunderland N, Skroblin P, Barwari T, et al. MicroRNA biomarkers and platelet reactivity: the clot thickens. Circ Res 2017;120:418-35.

19. Kannan M, Atreya C. Differential profiling of human red blood cells during storage for 52 selected microRNAs. Transfusion 2010;50:1581-8.

20. Doss JF, Corcoran DL, Jima DD, Telen MJ, Dave SS, Chi JT. A comprehensive joint analysis of the long and short RNA transcriptomes of human erythrocytes. BMC Genomics 2015;16:952.

21. Wang Z, Zhou D, Cao Y, et al. Characterization of microRNA expression profiles in blood and saliva using the Ion Personal Genome Machine $\left({ }^{\oplus}\right)$ System (Ion PGM System). Forensic Sci Int Genet 2016;20:140-6.

22. Bianchi E, Bulgarelli J, Ruberti S, et al. MYB controls erythroid versus megakaryocyte lineage fate decision through the miR-486-3p-mediated downregulation of MAF. Cell Death Differ 2015;22:1906-21.

23. Wang LS, Li L, Li L, et al. MicroRNA-486 regulates normal erythropoiesis and enhances growth and modulates drug response in CML progenitors. Blood 2015;125:1302-.

24. Bruning U, Cerone L, Neufeld Z, et al. MicroRNA-155 promotes resolution of hypoxia-inducible factor 1alpha activity during prolonged hypoxia. Mol Cell Biol 2011;31:4087-96.

25. Ivan M, Harris AL, Martelli F, Kulshreshtha R. Hypoxia response and microRNAs: no longer two separate worlds. J Cell Mol Med 2008;12: $1426-31$.
26. Romania P, Lulli V, Pelosi E, Biffoni M, Peschle C, Marziali G. MicroRNA 155 modulates megakaryopoiesis at progenitor and precursor level by targeting Ets-1 and Meis1 transcription factors. Br J Haematol 2008;143:570-80.

27. Yao M, Wang $\mathrm{X}$, Tang $\mathrm{Y}$, et al. Dicer mediating the expression of miR-143 and miR-155 regulates hexokinase II associated cellular response to hypoxia. Am J Physiol Lung Cell Mol Physiol 2014;307:L829-37.

28. Honda S, Arakawa S, Nishida Y, Yamaguchi H, Ishii E, Shimizu S. Ulk1-mediated Atg5-independent macroautophagy mediates elimination of mitochondria from embryonic reticulocytes. Nat Commun 2014;5: 4004.

29. O'Connell RM, Taganov KD, Boldin MP, Cheng G, Baltimore D.. MicroRNA-155 is induced during the macrophage inflammatory response. Proc Natl Acad Sci U.S.A 2007;104:1604-9.

30. Turner M, Vigorito E. Regulation of B- and T-cell differentiation by a single microRNA. Biochem Soc Trans 2008;36:531-3.

31. Zhu N, Zhang D, Chen S, et al. Endothelial enriched microRNAs regulate angiotensin II-induced endothelial inflammation and migration. Atherosclerosis 2011;215:286-93.

32. Pankratz F, Bemtgen X, Zeiser R, et al. MicroRNA-155 exerts cell-specific antiangiogenic but proarteriogenic effects during adaptive neovascularization. Circulation 2015;131:1575-89.

33. Trowbridge EA, Martin JF, Slater DN. Evidence for a theory of physical fragmentation of megakaryocytes, implying that all platelets are produced in the pulmonary circulation. Thromb Res 1982;28:461-75.

34. Slater DN, Trowbridge EA, Martin JF. The megakaryocyte in thrombocytopenia: a microscopic study which supports the theory that platelets are produced in the pulmonary circulation. Thromb Res 1983;31: 163-76.

35. Hudson JG, Bowen AL, Navia P, et al. The effect of high altitude on platelet counts, thrombopoietin and erythropoietin levels in young Bolivian airmen visiting the Andes. Int J Biometeorol 1999;43:85-90.

36. Lefrancais E, Ortiz-Munoz G, Caudrillier A, et al. The lung is a site of platelet biogenesis and a reservoir for haematopoietic progenitors. Nature 2017;544:105-9.

37. Caruso P, MacLean MR, Khanin R, et al. Dynamic changes in lung microRNA profiles during the development of pulmonary hypertension due to chronic hypoxia and monocrotaline. Arterioscler Thromb Vasc Biol 2010;30:716-23.

38. Ho JJ, Metcalf JL, Yan MS, et al. Functional importance of Dicer protein in the adaptive cellular response to hypoxia. J Biol Chem 2012;287: 29003-0.

39. Shen J, Xia W, Khotskaya YB, et al. EGFR modulates microRNA maturation in response to hypoxia through phosphorylation of AGO2. Nature 2013;497:383-7.

40. Li D, Ji L, Liu L, et al. Characterization of circulating microRNA expression in patients with a ventricular septal defect. PLoS One 2014;9: e106318.

41. Dickinson CJ. The aetiology of clubbing and hypertrophic osteoarthropathy. Eur J Clin Invest 1993;23:330-8. 